\title{
Amenaza, vulnerabilidad y riesgo ante eventos naturales. Factores socialmente construidos
}

\author{
Threat, risk and vulnerability to natural events. Socially constructed factors
}

\author{
Ana Cecilia Reyes Rivero ${ }^{1, *}$, Argenis de Jesús Montilla Pacheco ${ }^{2, \dagger}$, \\ Paola Germania Castillo García ${ }^{2, \ddagger}$, y Martha Narciza Zambrano Vera ${ }^{2, \otimes}$ \\ ${ }^{1}$ Universidad Pedagógica Experimental Libertador, Venezuela. \\ ${ }^{2}$ Universidad Laica Eloy Alfaro de Manabí, Ecuador. \\ \{anacereyes;argenismontilla\}@ hotmail.com, \{paolacastillo79;martha0558\}@gmail.com \\ Fecha de recepción: 25 de enero de 2017 — Fecha de aceptación: 17 de marzo de 2017
}

\begin{abstract}
Resumen-Al intentar explicar las razones por las cuales los desastres socionaturales traen consigo una cadena de nefastas consecuencias, se hace imperante analizar la concepción que se maneja del riesgo y de sus elementos asociados: amenaza y vulnerabilidad, dadas las discordancias, confusiones, vacíos e imprecisiones al respecto. Ello amerita de un análisis exhaustivo y del estudio de la multiplicidad de factores capaces de intervenir en su comprensión, y que de alguna forma pueden incidir en la carencia de una cultura preventiva. De allí emerge el presente ensayo, cuyo propósito es reflexionar sobre distintas concepciones del riesgo en diferentes momentos históricos, impregnándose del tipo de sociedad de donde provienen, y en la cual se originan modelos teóricos como abstracciones de una realidad construida en el imaginario social, que intenta explicarlas y comprenderlas. Se trata entonces de esquemas que demandan una transformación a través de la educación, pues es ésta la herramienta más idónea y al mismo tiempo capaz de influir y modificar la manera de percibir, valorar y actuar ante fenómenos naturales adversos y sobre aquellos factores que determinan su conversión en eventos calamitosos; todo con la finalidad de que se interpreten global y sistémicamente, redundando en una preparación afectiva y efectiva, que posibilitará la actuación acertada ante una situación de riesgo o peligro.
\end{abstract}

Palabras Clave-Riesgo, Amenaza, Vulnerabilidad, Evento adverso, Imaginario social.

\begin{abstract}
In attempting to explain the reasons why socio-natural disasters bring with them a chain of disastrous consequences, it is imperative to analyze the conception of risk and its associated elements: threat and vulnerability, given the discordances, confusions, gaps and inaccuracies about it. This requires a thorough analysis and the study of the multiplicity of factors capable of intervening in its compression, and that may somehow affect the lack of a Preventive Culture. From this emerges the present essay, whose purpose is to reflect on different conceptions of risk in different historical moments, impregnating of the type of society from which they originate, and in which theoretical models originate as abstractions of a reality constructed in the social imaginary, which attempts to explain and understand them. These are then schemes that merit a transformation through education, since this is the most appropriate tool, and at the same time able to influence and modify the way of perceiving, valuing and acting against adverse natural phenomena, and those factors that determine their conversion into calamitous events; All with the aim of being interpreted globally and systemically, redundating in affective and effective preparation, which will enable the understanding and correct action in a situation of risk or danger.
\end{abstract}

Keywords-Risk, Threat, Vulnerability, Adverse event, Social imaginary.

\section{INTRODUCCIÓN}

$\mathbf{L}$ a carencia de una cultura preventiva ante desastres socionaturales es una realidad innegable que genera e incluso aumenta la vulnerabilidad en las comunidades, con consecuentes riesgos de dimensiones extraordinarias que pueden producir pérdidas humanas y materiales incalculables. De allí la necesidad de explicar cómo las apreciaciones que giran alrededor del riesgo son influenciadas y están determinadas por diversas percepciones Mazzitelli and Aparicio (2010), Cid Ortiz et al. (2012), Osorio Yepes and Díaz Facio Lince (2012), Ramírez and Ofelmina (2015), tanto individuales como colectivas en torno al mismo y que se expresan mediante representaciones y más complejamente a través de los ima-

\footnotetext{
* Docente Investigador.

†Doctor en Ecología Tropical.

${ }^{\ddagger}$ Licenciada en Contaduría y Auditoria, Magíster en Tributación Y Finanzas.

${ }^{\otimes}$ Profesora de Segunda Enseñanza.
}

ginarios sociales (Curiel, 2012). (Federación Internacional de Sociedades de la Cruz Roja y de la Media Luna Roja, 2015).

Es evidente entonces, que al intentar buscar una explicación del porqué los desastres socionaturales traen consigo una cadena de consecuencias casi imposibles de controlar, se hace imperante analizar cuál es la concepción del riesgo y de sus elementos asociados que a lo largo del tiempo se maneja, específicamente la amenaza y la vulnerabilidad, dadas las discordancias, confusiones e imprecisiones existentes en relación a estas definiciones, por lo que se precisa de la ejecución del análisis exhaustivo de dichos términos, así como de la multiplicidad de factores de diversa índole, capaces de intervenir en su compresión, y que de alguna manera pudieran incidir en la carencia de una Cultura Preventiva.

Esta es la razón por la cual se presenta el siguiente ensayo, cuyo propósito es reflexionar sobre las distintas concepciones y contenidos que se tienen del riesgo y su relación dialéctica con la amenaza y la vulnerabilidad, pues éstas varían en diferentes momentos históricos, en función a las condiciones 
de la sociedad de la que provienen y en donde se originan modelos teóricos, vistos éstos como abstracciones de una realidad construida en el imaginario popular, que intenta explicarlas y comprenderlas.

De igual forma, se expone sobre algunos de los múltiples factores periféricos responsables, que condicionan los procesos de análisis, comprensión y asimilación del riesgo, la amenaza y la vulnerabilidad, capaces de intervenir y bloquear a los individuos, para que no exista una completa compresión del problema, con implicaciones en aspectos naturales, materiales y cognitivos, convirtiéndose en esquemas mentales a través de los que determinados grupos humanos expresan su forma de concebir el riesgo.

De allí que resulte imperante la transformación de estos esquemas y modelos a través de la educación, formadora por excelencia y única herramienta capaz de influir en el individuo, tanto en su manera de percibir, como de valorar y actuar ante la realidad de los fenómenos naturales adversos, y especialmente sobre aquellos factores que determinan la transformación de los mismos en eventos calamitosos; todo ello con la finalidad de que sean interpretados de una manera global y sistémica, que redunde en una preparación afectiva y efectiva, que permita comprender y actuar acertadamente ante una situación de riesgo o peligro.

\section{Metodología}

La investigación que se llevó a cabo fue de carácter analítica reflexiva, pues consistió en analizar las opiniones que diferentes autores manejan en relación al tema del riesgo, la amenaza y vulnerabilidad, pero además de la percepción social que de éste se tiene. En tal sentido, se precisó la consulta de distintas fuentes bibliográficas especializadas en la temática abordada, fundamentalmente libros y artículos publicados en revistas científicas.

Especial cuidado se adoptó en la selección de los trabajos revisados, considerando que la mayoría de ellos sean de reciente publicación, pues en asuntos de análisis de riesgo y sus elementos asociados como vulnerabilidad y amenaza se producen cambios conceptuales con importante frecuencia que inciden en la forma de interpretarlos. En consecuencia, el arqueo bibliográfico realizado permitió acceder al dominio conceptual actualizado, y explicar los fundamentos teóricos y metodológicos que actualmente se manejan sobre el riesgo y la percepción social del mismo.

Partiendo del hecho que muchos autores manejan indistintamente los conceptos de riesgo, vulnerabilidad y amenaza, y a los efectos de definir en forma precisa lo que implica cada uno, se hizo énfasis en aclarar que hay una marcada diferencia entre cada uno de ellos, y que aunque suelen estar inmersos en un mismo escenario, son en esencia distintos, ello lo asevera Lavell (2006) cuando sostiene que "a pesar de la clara diferencia entre una y otra noción, son, sin embargo, cercanamente relacionadas e interrelacionadas de forma jerárquica o concatenada, y el cabal entendimiento y distinción entre ellas facilita de forma importante la construcción de opciones de intervención en la temática del riesgo y desastre" (p.1).

\section{Amenaza, vulnerabilidad y riesgos: una posible relación dialéctica}

De acuerdo a Jeréz Ramirez (2014), las definiciones de riesgo usualmente aceptadas son entendidas sobre la base de un esquema lógico que indica la relación entre un suceso dañino y unas víctimas afectadas, relación que proviene de una fórmula muy conocida que señala al evento agresor como la amenaza (A), y la susceptibilidad o tendencia de la población a sufrir el impacto, es decir, la vulnerabilidad (V). De allí que se calcule el riesgo como: Riesgo $=$ Amenaza $\times$ Vulnerabilidad $=$ Amenaza + Vulnerabilidad.

Con ello se pudiera entender, que al aplicar estas fórmulas, y planteando casos hipotéticos se llegue a suponer, que no hay riesgo sin amenaza o cuando la vulnerabilidad sea cero; o que siendo la población vulnerable, el riesgo sea cero, si no existe amenaza potencial. Supuestos que son completamente irreales, por cuanto el riesgo permanecerá latente en una mayor o menor proporción, dado que los peligros naturales siempre van a existir (Oficina de las Naciones Unidas para la Reducción del Riesgo y el Desastre, 2015), siendo los tipos de amenazas y su magnitud, dependientes de la realidad natural de cada región; mucho más si se trata de la vulnerabilidad de una población que pudiera estar influida por una multiplicidad de factores difícilmente cuantificables. Ello quiere decir que un razonamiento que aparentemente resulta lógico y correcto, induce a error, en virtud de que se trabaja con realidades naturales y sociales inexactas y falibles.

La gran debilidad de esta definición es que promueve únicamente elementos matemáticos, señalando a las amenazas y las vulnerabilidades como factores que solo se suman o se multiplican, lo cual no es suficiente. Sin embargo ha resultado de gran valor por cuanto significa un claro avance, en el sentido amplio que ésta pretende, al interpretar que no todo lo que ocurre depende del fenómeno agresor, pues la población involucrada, también tiene un papel fundamental, de ella depende que se favorezcan o se dificulten los efectos. Siendo así, se considera que el riesgo no pueda reducirse a cero por cuanto es imperante reconocer las amenazas socionaturales, que irremediablemente incluye al individuo y su comportamiento en colectivo.

Caram and Pérez (2006), refieren sobre cuatro elementos que dan cuenta a la complejidad del riesgo, visibles analíticamente, pero inexorablemente vinculados entre sí, éstos son:

1. La peligrosidad, que no es más que el potencial de amenaza de cualquier fenómeno natural adverso, por ello, mientras más información se tenga sobre éste, mayores posibilidades hay de predecir su ocurrencia en aquellos casos donde la predicción es posible.

2. La exposición, que hace mención a las construcciones materiales y a la distribución de la población, puesta de manifiesto en las precarias edificaciones, carencia de servicios, construcción en áreas inestables, que en conjunto constituyen la combinación de elementos materiales, infraestructura y población potencialmente afectadas ante un evento adverso.

3. La vulnerabilidad, definida como la situación en la que se encuentra la población, características o atributos 
existentes en la misma, que le permiten o le imposibilitan enfrentar un imprevisto fenómeno natural; y finalmente,

4. la incertidumbre ante esos eventos, que impone la necesidad de involucrar en la toma de decisiones a quienes están o estarán expuestos al peligro, haciéndose necesario el conocimiento de las percepciones del riesgo de los actores sociales.

De lo expresado, se desprende que el análisis de un fenómeno natural adverso y sus consecuencias va mucho más allá de la amenaza o el evento (Jeréz Ramirez, 2014), por cuanto se integran distintas dimensiones; de allí que a la hora de planificar y diseñar obras de infraestructura y de protección, es importante definir "riesgos aceptables" al considerar el elemento social. Con ello no se está excluyendo la posibilidad de que el riesgo no pueda calcularse, pero es necesario dejar claro que su estimación es relativa, y por ende los indicadores proporcionan "riesgos relativos," que son pautas que sí permitirán tomar decisiones y definir prioridades de prevención y mitigación.

Para evitar caer en dichos errores de interpretación, se asume que el concepto de riesgo implica una relación dialéctica entre amenaza y vulnerabilidad y ambas al integrarse en un mismo proceso generan un nivel distinto de realidad, no limitado a una interacción entre factores, como normalmente se suele entender. De allí que Lavell (1999), denote a la amenaza, la vulnerabilidad y el riesgo como "factores socialmente construidos" (p.7), en razón de que los eventos físicos extremos asociados con procesos naturales pueden transformarse en amenazas, solamente por intermediación humana; lo mismo ocurre con la vulnerabilidad, su aumento o disminución dependerá de las conductas individuales y colectivas.

Al respecto, Cardona (2003), explica como estos términos fueron utilizados por mucho tiempo sin que se tuviera suficiente claridad sobre ellos, relacionándolos a una posibilidad y a un hecho, es decir, vinculándolos tan solo a una causa: la amenaza natural, ante la cual no hay mucho que hacer, entre otras cosas, porque son fenómenos que no pueden ser controlados y en la mayoría de los casos imposibles de predecir. Incluso, se hizo uso del término riesgo a lo que hoy es entendido como amenaza, y se utilizó el vocablo vulnerabilidad, asignándole el mismo significado de riesgo.

Otro de los aspectos que han generado imprecisiones en la terminología referida es el uso que se le ha dado al asociarla con ámbitos distintos, es así como la vulnerabilidad se ha relacionado con condiciones de desventaja: ancianos, pequeños o mujeres; factores que también han incidido para que no exista una completa compresión y claridad de estos conceptos (Aguirre, 2004), cuya distinción facilitará el entendimiento del riesgo, lo cual permitirá reducirlo o mitigarlo.

Señala Cardona (2003), que poco a poco se entendió que la vulnerabilidad es más compleja de lo que se creía, comenzó a verse como "una predisposición intrínseca a ser afectado o a ser susceptible a sufrir daños", como la "susceptibilidad física, económica, política o social" (p.12), expresada como la factibilidad de que un individuo o sistema social se afecte por un fenómeno desestabilizador, que en este caso es la amenaza natural. De esta manera, el riesgo pudo expresarse en forma matemática, resultante, según lo expone el autor, “... de la convolución de la amenaza y la vulnerabilidad” (p.12).

En el marco de las observaciones anteriores, resulta oportuno definir la amenaza como un factor externo, como la probabilidad de ocurrencia de un evento natural en un tiempo y lugar determinado (Centro de Coordinación para la Prevención de los Desastres Naturales en América Central., 2017). En efecto, Cardona et al. (2006), señalan que las amenazas obedecen a fenómenos naturales que representan peligros potenciales y latentes.

Sobre el mismo particular Escobar-Gómez (2007), aduce que una amenaza se considera como tal, cuando pudiera afectar directa o indirectamente la vida o los bienes de una población, ocasionada por un fenómeno natural o provocada por el hombre, que actúa en un tiempo y lugar específico. Interpretándose de esta forma, no se limita exclusivamente a factores naturales sino que también adquiere un carácter social, de allí que la amenaza debe ser entendida como el evento natural extremo que representa un peligro potencial inherente a los propios fenómenos naturales o eventos adversos (Oficina de los Estados Unidos de Asistencia para Desastres en el Extranjero, 2014).

Por esa razón Briones-Gamboa (2007) clasifica a las amenazas en: naturales (volcanes, terremotos, huracanes, entre otros), antrópicas a las que también denota como tecnológicas (uso inadecuado de plaguicidas, guerras y otras), y socionaturales (destrucción y degradación de cuencas, contaminación sónica, hídrica, atmosférica, etc.).

En ese mismo sentido Lavell (1999), realiza una diferenciación entre amenazas naturales y amenazas socionaturales, por lo que define a las primeras como aquellos eventos que por su propia naturaleza en un determinado momento se convierten en un peligro para el hombre, y se ilustra con el ejemplo de un movimiento sísmico que se produzca en un desierto en el que no existe riesgo alguno porque no hay exposición de personas o bienes materiales, si se compara con un sismo de menor intensidad pero en el casco urbano de una ciudad, que podría destruir inmuebles y acarrear la muerte de miles de personas.

En lo que respecta a las amenazas socionaturales, se entiende que sobre los procesos naturales interfiere el hombre, que según Lavell (1999), "son aquellos eventos que se gestan en la intersección de la sociedad con los procesos de la naturaleza" (p.8), de una forma que rebasan el potencial autorregulador del sistema, por lo que traspasan la capacidad de ajuste, flexibilidad de sus componentes para absorber, asimilar y/o neutralizar tales perturbaciones, desequilibrándose irreversiblemente. En tal sentido Andrade and Laporta (2009), señalan que un fenómeno solo adquiere la condición de peligroso y consecuencialmente pasa a ser parte del riesgo, cuando su ocurrencia se produce o se pronostica en un territorio ocupado por una determinada comunidad. La causa es el hombre como depredador del entorno, haciendo que los eventos naturales se conviertan en desastrosos o calamitosos con efectos adversos sobre él mismo, sus bienes y el ambiente.

Sobre la base de las consideraciones anteriores, resulta oportuno definir desastre, palabra que proviene de dos términos de origen latino: Di, Dis que es igual a Des y Astrum que significa Astro, Hado; etimológicamente expresa "desgracia, calamidad, 
suceso infausto lamentable”. Según lo refiere Curiel (2012), un desastre resulta de la combinación de amenazas en situaciones de vulnerabilidad asociadas a la insuficiente capacidad para mitigar los potenciales efectos del riesgo.

Como bien se ha explicado, un fenómeno natural en sí mismo no constituye un desastre natural, éste actúa solo como el detonante de las condiciones de una determinada población, donde confluyen una serie de factores, entre ellos, los socioeconómicos como la pobreza, las concentraciones humanas y la degradación ambiental, los cuales serán variables dependiendo de cada realidad.

Para ilustrarlo, Lavell (1999), refiere la existencia de cinco contextos particulares que permiten comprender la vulnerabilidad presente en el medio urbano específicamente, y que por ende se convierten en causantes de desastres, éstos son: (a) la concentración, la densidad y la centralización, (b) la complejidad y la interconectividad de la ciudad, (c) la ciudad informal o la ciudad de campesinos, (d) la degradación ambiental urbana y la vulnerabilidad estructural y, (e) la vulnerabilidad política e institucional; factores que en sí mismos son heterogéneos, distintos en cada ciudad, pudiendo estar acentuados unos más que otros, convirtiéndose en causantes de desastres en distinta proporción, según sea el ámbito donde se desarrollen.

Las primeras explicaciones se fundaron sobre la base "de un límite de daños asociados de carácter cuantitativo y universal", por ejemplo, cifras de pérdidas en dólares, número de personas fallecidas, heridas o desaparecidas, cantidad de individuos damnificados, todos valores numéricos; elementos éstos que pudiera llevar a consideraciones pocos objetivas, como excluir de esta categoría a un evento menos significativo porque la magnitud de pérdidas fueron mínimas si se compara con la superficie afectada, y catalogar como desastre a un acontecimiento derivado en una localidad pequeña aunque el número de muertes y pérdidas no alcance las estimaciones previstas. Para evitar caer en equivocaciones, se plantea la necesidad de definir a los desastres en función de la vulnerabilidad de las sociedades.

Como ya se ha indicado, en el cálculo del riesgo el otro elemento es la vulnerabilidad comprendida como el factor interno, que va a depender de las condiciones que una región posea para enfrentar esa amenaza, condiciones que serán mayor o menor en la medida que existan debilidades de tipo social, económica y estructural entre otras, capaces de ocasionar daños cuantiosos, y en algunos casos irreparables. En consecuencia, la vulnerabilidad permitirá determinar la propensión que tienen los elementos de que puedan ser perturbados o de tolerar pérdidas ante una amenaza específica; razón por la cual Lavell (1999), la define como la tendencia a sufrir daños y a la vez la medida de las dificultades que enfrenta una comunidad para recuperarse de las consecuencias, expresada en condiciones de inseguridad, producto de procesos sociales íntimamente relacionados con las modalidades de desarrollo o no desarrollo.

Otra definición de vulnerabilidad es la imposibilidad de un grupo social para responder a cambios por efectos de un evento adverso, bien sea por su incapacidad, su estado de exposición o su susceptibilidad (Briones-Gamboa, 2007). De la misma forma Andrade and Laporta (2009), expresan que si bien es cierto que la vulnerabilidad es la medida de exposición de un individuo o un colectivo ante los efectos de un fenómeno adverso, desde esta óptica sólo es posible desarrollar una medida cuantificable de lo que sería la vulnerabilidad, orientada a la probabilidad de ocurrencia del evento con una determinada "intensidad, frecuencia y duración" (p.4), lo que induce al cálculo de los efectos desde el punto de vista numérico; de allí la importancia de que en el estudio de la vulnerabilidad, éste se relacione más con la peligrosidad inherente al fenómeno, que con el fenómeno mismo, por cuanto se afectan a grupos sociales, y su heterogeneidad es lo que determina, en mayor parte, las consecuencias catastróficas de la peligrosidad.

De allí surge la explicación del porqué existe una obligatoria relación entre la amenaza y la vulnerabilidad, que aun siendo factores con características y especificidades sumamente heterogéneas y que a los fines de analizarlos, no es posible hablar de amenaza sin referirse a vulnerabilidad y viceversa. Debe haber una tendencia o propensión a sufrir daños, para que un evento físico, natural, social o tecnológico se convierta en amenaza con repercusiones que desencadene en un desastre, sino es simplemente un evento; es decir, existe una relación, dialéctica, lógica y dinámica entre ambos agentes.

Ahora bien, si se pretende una estimación del riesgo, es preciso realizar una evaluación de la amenaza natural pudiendo ser ésta predecible o no; y en lo que respecta a la vulnerabilidad, deben ser considerados aspectos técnicos, que son fácilmente cuantificables, en términos físicos y funcionales (infraestructura, líneas de vida, por ejemplo) y aquellos de carácter social, aun más importante, que deben valorarse en términos cualitativos, mucho más complejos en función del contexto social y cultural.

De acuerdo con los razonamientos planteados, resulta oportuna la definición de riesgo, realizada por Cardona et al. (2006), de la siguiente manera:

“... es un concepto extraño, representa algo irreal, en tanto que está siempre relacionado con azar, con posibilidad, con algo que aún no ha sucedido. Es una abstracción de un proceso de transformación que denota simultáneamente posibilidad y realidad. Es algo imaginario y escurridizo que parece sólo existir en el futuro y que refleja un estado indeseable de realidad, pero su existencia compleja es consustancial al hombre" (p.1).

Precisando de una vez, el riesgo es el daño que se espera, producto de la relación o coexistencia entre la probabilidad de ocurrencia de eventos peligrosos y de los elementos expuestos a tales amenazas, es el resultado que se calcula en función de una amenaza específica y las condiciones de vulnerabilidad que esa región posea para enfrentarlas. De allí que en la medida del riesgo convergen todo un agregado de factores que deben ser vistos de manera holística, en donde cada una de las disciplinas o ciencias que se encargan de su estudio deberían jugar un papel fundamental en esa integralidad, muy lejos de la visión atomista con la que se pretende analizar el riesgo hasta ahora.

Al respecto Briones-Gamboa (2007), hace una reseña de la evolución histórica de este término, el cual se encuentra ligado con el tiempo "... el pasado no implica riesgo, sólo el futuro" (p.7). Es decir, se asocia con la idea de porvenir sin certeza y esta incertidumbre es lo que obliga la necesidad de predecir, 
como una vía para reducir los temores. Agrega dicho autor que el concepto de riesgo es relativamente reciente, tiene sus orígenes a finales de la Edad Media, y que anteriormente se le denominaba peligro y era imposible mitigar; en las sociedades antiguas lo que dominaba era el peligro, asociado con algo concreto, mientras que en las modernas es el riesgo y se considera el margen de incertidumbre sobre el posible daño.

Es así como al vocablo riesgo, se integraron dos elementos: la probabilidad y la decisión, y la idea de calcular la probabilidad implica aceptar un margen de elección personal, pero la probabilidad como atributo de riesgo tiene una perspectiva económica y éste no sólo puede ser tomado como un concepto estadístico. Expone Briones-Gamboa (2007), que los científicos y expertos para calcular el riesgo usan fórmulas estadísticas basadas en la probabilidad según el impacto negativo de eventos peligrosos pasados, tratando el comportamiento de la gente ante los riesgos como irracional; mientras que los sociólogos, critican la idea de riesgo objetivo, arguyendo que las percepciones de riesgo no son irracionales y deben ser vistas como juicios bajo la incertidumbre.

En este sentido Cardona (2003), describe la concepción que se le ha dado al riesgo y las terminologías asociadas desde distintas perspectivas disciplinares. Es así como desde el enfoque de las Ciencias Naturales, los desastres naturales son vistos sólo como fenómenos físicos, en virtud de que el interés sobre este tema es principalmente de geofísicos, sismólogos, meteorólogos, geólogos, entre otros. El hecho de que estos fenómenos que en su mayoría no se pueden predecir con certeza y precisión a pesar de los avances tecnológicos, deriva que desde esta perspectiva, la producción de daños, pérdidas humanas y materiales sean tratadas como consecuencias inevitables.

Por otro lado, las Ciencias Aplicadas se centran en los efectos del suceso, asociándolos con la amenaza que pudiera generar en los sistemas estructurales, entendiéndose a la vulnerabilidad como la susceptibilidad de sufrir daños, producto de la capacidad estructural; al riesgo, como el potencial de pérdidas según los daños factibles y; al desastre, como las consecuencias o el impacto que se causa sobre la sociedad que sufre estos daños y pérdidas.

No cabe duda, que el aporte de estas ciencias al estudiar la capacidad de los elementos estructurales o sistemas expuestos con el fin de estimar los daños físicos, significó un cambio de paradigma en lo que se refiere al riesgo, sin embargo, aunque se propone un concepto del mismo mucho más completo, el enfoque sigue siendo parcial y reduccionista, en razón de que en la práctica la evaluación de la vulnerabilidad física, sigue sustituyendo la evaluación del riesgo, el cual se deja como un resultado secundario. En síntesis, existe un desconocimiento de los aspectos sociales, culturales, económicos y políticos que deben reflejarse en la estimación de la vulnerabilidad y el riesgo.

Desde el enfoque de las Ciencias Sociales, Cardona (2003) expone que el riesgo es coligado al estudio sobre las reacciones, la percepción individual y colectiva, así como a las respuestas de la población ante emergencias. Esta perspectiva señala que los desastres no son sinónimos de eventos naturales, por cuanto no están referidos únicamente al daño físico potencial o a determinantes demográficas, éstos ocurren solo cuando las pérdidas que se producen por un suceso, superan la capacidad de la población de soportarlas o cuando los efectos impiden recuperarse fácilmente; de allí que la vulnerabilidad no pueda ser definida o medida sin hacer referencia a la capacidad de la población de absorber, responder y recuperarse del impacto del suceso.

Adicionalmente, la vulnerabilidad es interpretada como una "característica" o "propiedad" y no como una condición, circunstancia o predisposición resultante de una fragilidad, hecho que hace perder de vista la amenaza que por supuesto es muy importante por cuanto es el fenómeno detonante. Este enfoque aborda el concepto de riesgo considerando que la vulnerabilidad e inclusive la amenaza son el resultado de procesos sociales, económicos y políticos.

En razón a todo lo anterior, Cardona (2003), expone sobre la necesidad de que la vulnerabilidad y el riesgo sean vistos desde una perspectiva holística, denotando al riesgo como un concepto complejo, alrededor del cual confluyen muchos factores a la vez, tres de ellos fundamentales a la hora de estimarlo: la eventualidad, las consecuencias y el contexto, siendo este último fundamental al momento de tomar cualquier decisión, por cuanto el ámbito en el que se produzca un hecho, que pudiera convertirse en amenaza, es muy particular, los actores involucrados son diversos y por ende la capacidad de gestión debe ser específica para cada realidad.

Según se ha visto, el dimensionamiento del riesgo debe entenderse de una manera integral y holística, como un todo complejo sistémico, donde cada uno de los factores capaces de influir, bien sean de tipo social, organizacional e institucional deben ser considerados. Ello implica aceptar que las vulnerabilidades van mucho más allá de la incidencia de agentes externos, de los elementos físicos como el ordenamiento territorial, las infraestructuras o las líneas de vida, siendo además fundamental considerar los agentes internos, quizás los más importantes, relativos a las fragilidades sociales, expresados en el conocimiento, la comunicación y la preparación que tengan los grupos sociales para soportar el impacto de un evento socionatural y sus implicaciones.

Por otra parte, el análisis del riesgo siempre está vinculado a una decisión, de allí que éste es consecuencia de una decisión racional, y renunciar al riesgo es renunciar a la racionalidad, el riesgo está en función de una determinación y el peligro del entorno, es decir, el posible daño será consecuencia del fallo a que diera lugar. Es por ello que los resultados producto de estas acciones, en algunos casos pudieran ser positivos, pero en otros casos nefastos.

\section{Conclusiones}

De acuerdo con los razonamientos desarrollados, resulta fundamental el verdadero entendimiento del riesgo, lo que orientará acertadamente la ejecución de acciones, cualquiera que sean, desde las menos significativas hasta las más complejas, aquellas que deban tomarse a corto, mediano o largo plazo; y esta comprensión del riesgo debe ser previa a la decisión sobre las determinaciones que han de tomarse, evaluación que involucra elementos diversos y complejos, que como 
indica Cardona (2003), “...tocan raíces de la sociedad, el conocimiento, los valores, las emociones e incluso la propia existencia" (p.11). En dicho propósito, resulta fundamental conocer cuáles son las percepciones individuales y colectivas en torno al riesgo, qué valores, pensamientos y conductas lo han reproducido y construido, cómo éste se concibe en el imaginario colectivo y en consecuencia representado socialmente.

Respecto a lo anterior, Ruiz Guadalajara (2005), expone que de acuerdo con la antropología del riesgo, los desastres entendidos como procesos y no como eventos explosivos, no son más que complejos históricos de acumulación progresiva de vulnerabilidades, producto de la acción de amenazas naturales y sociales, con el agregado de nuevas amenazas que subsecuencialmente se incorporan al proceso, por la acción de la construcción social del riesgo. Es así como grupos sociales en sus prácticas y representaciones, al aumentar sus vulnerabilidades construyen progresivamente el riesgo, lo que implica desastres en potencia o en vías de realización.

Ello refuerza la conclusión a la que han llegado estudios realizados en materia de riesgos, donde se alude que la vulnerabilidad es imposible eliminarla totalmente, pues nada más puede transformarse, acumularse o disminuirse, postura que concuerda con la opinión dada por Ruiz Guadalajara (2005), cuando expresa “... la sociedad libre de riesgos o el hombre emancipado de las amenazas sólo existe como argumento de utopías..." (p.3).

En efecto, el riesgo en relación con la problemática de los desastres, es una condición latente que repercute en posibles daños futuros y pérdidas en un determinado grupo social, y en razón de que es el producto de la compleja relación dialéctica de las particulares condiciones físicas y las características de vulnerabilidad, el mismo es dinámico y cambiante. Éste es siempre el resultado de las acciones individuales, sociales, organizacionales o institucionales, conscientes e inconscientes; por ello es preciso afirmar que el riesgo es una construcción social.

En definitiva, resulta que los contenidos y concepciones del riesgo en los diferentes momentos históricos, se encuentran impregnados por el tipo de sociedad de la cual emanan, lo que origina modelos teóricos que a su vez son abstracciones de la realidad para intentar explicarla y comprenderla. Por ello, no es fortuito que actualmente se vinculen las nociones de riesgo y desastre, dado que ambas son el producto de la interacción entre la cultura y la experiencia social; por tanto, su compresión requiere de un enfoque integral u holístico que relacione estructural y funcionalmente todos los componentes, elementos y fenómenos de la sociedad con el entorno natural y aquel que ha sido construido.

Resulta evidente, que el riesgo al ser un proceso socialmente construido tiene la posibilidad de ser manejado, por ende, modificado y transformado, para así reducir sus fuentes generativas. De allí el reconocimiento urgente de la educación como único medio para potenciar cambios profundos individual y colectivamente, como la llave instrumental capaz de lograr el accionar que se espera y que traerá consigo sociedades conscientes de su realidad natural, reflexivas, desarrolladas y seguras.

Ello significa trabajar para producir cambios sustanciales en los sistemas sociales, políticos, económicos, culturales y en la organización de las comunidades, lo que iría en correspondencia con nuevas formas de observar el mundo en su dinámica sujeto-objeto-suceso. En este propósito, el reto debe ser educar para transformar valores, hábitos, conductas e ideas, lo que consecuencialmente implica, tal como fue expuesto por el filósofo Sartre, citado por Vargas Hernández (2007) “... un proceso de construcción, destrucción y reconstrucción” hacia una cultura de la prevención.

\section{AgradeCimientos}

La realización de este trabajo fue posible gracias a la colaboración prestada por La Universidad Pedagógica Experimental Libertador - Instituto Pedagógico de Barquisimeto y por la Universidad Laica Eloy Alfaro de Manabí, Facultad de Hotelería y Turismo.

\section{Bibliografía}

Aguirre, B. E. (2004). Los desastres en latinoamérica: vulnerabilidad y resistencia. Revista mexicana de sociología, 66(3):485-510.

Andrade, M. I. and Laporta, P. (2009). La teoría social del riesgo: Una primera aproximación a la vulnerabilidad social de los productores agropecuarios del sudoeste bonaerense ante eventos climáticos adversos. Mundo agrario, 10(19):00-00.

Briones-Gamboa, F. (2007). La complejidad del riesgo: breve análisis transversal. Revista de la Universidad Cristóbal Colón, 20(3):9-19.

Caram, M. and Pérez, S. A. (2006). Entre el riesgo ambiental y el riesgo social: buscando una salida a la tenencia irregular. Revista argentina de sociología, 4(6):50-64.

Cardona, A., Darío, O., et al. (2006). "midiendo lo inmedible" indicadores de vulnerabilidad y riesgo.

Cardona, O. D. (2003). La necesidad de repensar de manera holística los conceptos de vulnerabilidad y riesgo. una crítica necesaria para la gestión.

Centro de Coordinación para la Prevención de los Desastres Naturales en América Central. (2017). Ciudad de Guatemala.

Cid Ortiz, G., Castro Correa, C., and Rugiero de Souza, V. (2012). Percepción del riesgo en relación con capacidades de autoprotección y autogestión, como elementos relevantes en la reducción de la vulnerabilidad en la ciudad de la serena. Revista Invi, 27(75):105-142.

Curiel, M. (2012). Un compendio sobre el estudio de las representaciones sociales. valencia. Revista ciencias de la educación, 22(39):237-254.

Escobar-Gómez, A. A. (2007). Percepción de los riesgos y desastres. Caso aldea Shalaguá, municipio de Camotán, Chiquimula . page 123 .

Federación Internacional de Sociedades de la Cruz Roja y de la Media Luna Roja (2015).

Jeréz Ramirez, D. O. (2014). Prevención y mitigación de desastres en Colombia: Racionalidad comunicativa en políticas públicas.

Lavell, A. (1999). Gestión de riesgos ambientales urbanos. Red de Estudios Sociales en Prevención de Desastres en América Latina, www. desenredando. org. 
Lavell, A. (2006). Consideraciones en torno al enfoque, los conceptos y los términos que rigen con referencia a la reducción del riesgo y la atención de desastres en los países andinos miembros del caprade. Recuperado de: http://www. comunidadandina. org/predecan/doc/rl/docAllan1. pdf.

Mazzitelli, C. A. and Aparicio, M. (2010). El abordaje del conocimiento cotidiano desde la teorãa de las representaciones sociales. Revista Eureka sobre Ense $\tilde{A} \pm$ anza y Divulgaci $\tilde{A}^{3} n$ de las Ciencias, 7(3).

Oficina de las Naciones Unidas para la Reducción del Riesgo y el Desastre (2015). Informe de evaluación global sobre reducción del riesgo de desastres.

Oficina de los Estados Unidos de Asistencia para Desastres en el Extranjero (2014). Oficina de los Estados Unidos de Asistencia para Desastres en el Extranjero (USAID). Reducción del Riesgo de Desastre a Nivel Local. Memoria del I Foro Latinoamericano y del Caribe de Reducción del Riesgo de Desastre a Nivel Local. 2014.

Osorio Yepes, C. D. and Díaz Facio Lince, V. E. (2012). Modelos de intervención psicosocial en situaciones de desastre por fenómeno natural. Revista de Psicología Universidad de Antioquia, 4(2):65-84.

Ramírez, J. and Ofelmina, D. (2015). Construcción social del riesgo de desastres: la teoría de representaciones sociales y el enfoque social en el estudio de problemáticas socioambientales.

Ruiz Guadalajara, J. C. (2005). De la construcción social del riesgo a la manifestación del desastre: Reflexiones en torno al imperio de la vulnerabilidad. Desacatos, (19):99-110.

Vargas Hernández, J. (2007). La culturocracia organizacional en méxico, edición electrónica gratuita. Texto completo en www. eumed. net/libros/2007b/301. 